a lingering soreness in the tibia, especially if accompanied by pain and stiffness in the ankle-joint, may, in view of the facts here enumerated, find its explanation in the existence of this peculiar fracture.

\title{
ON THE TREATMENT OF THE CHANCROID.
}

BY F. B. GREEOUGH, M. D.,

Physician of the Department for Skin and Venereal Diseases at the Boston Dispensary, and Clinical Instructor in Venereal Diseases in the Harvard Medical School.

I AM aware that the subject of this article will seem to many of little interest and of slight importance, but as chancroids are by no means uncommon, and, except in the large medical centres, must come under the treatment of the general practitioner, and as the conclusions to which my experience in treating a large number of cases forces me are diametrically opposed to those generally received, taught, and practiced, and moreover as these conclusions, if correct, will save patients considerable pain, not to mention other advantages to be referred to hereafter, I have thought that perhaps a small part of the space in the Journar might for once be taken from matters of more general interest.

By chancroid I mean the soft chancre as distinguished from the hard or indurated, the local venereal ulcer, or simply the chancre, as the modern German school has named it, arguing, with justice, that the term chancre, even when qualified by the adjective inclurated, is not properly applicable to the primary syphilitic lesion, as this often consists simply of an indurated papule with the slightest erosion, and sometimes of the induration alone, with absolutely no lesion of continuity.

This ulcer of which we are treating has certain well-marked characteristics; it always results from direct contagion from an ulcer of the same nature, the only factor necessary for its genesis being the absorption of some of the secretion of such an ulcer by the skin or mucous membrane; it has an apparent period of incubation, varying from a few hours to several days; it begins as a small pustule, which is soon broken, and the base of which is seen to consist of a minute ulcer. This gradnally spreads, circularly or elliptically when on a smooth surface, but is apt to follow the direction of any depression or fold in which it may happen to be situated, as in the sulcus at the corona, or in a fold of the mucous membrane of the anus; its edges are sharp cut, as if with a punch, and its base is covered with a dirty, yellowish-gray, pultaceous deposit which cannot be wiped off; and it secretes a purulent fluid which, inoculated on the patient or on another person, will reproduce an ulcer of the same sort. Having gone on increasing in size, more or less, according to circumstances, but usually not extending as deep 
as the subcutaneous tissue, for the space of from four to six weeks, the base begins to clean up, and gradually takes on the appearance of a healthy granulating wound; the spreading at the periphery stops, and then the process of healing begins and proceeds exactly like that of any lesion of continuity of the skin caused by a burn or cut, and results in a cicatrix of fibrous tissue such as is always formed when the matrix of the skin, that is, the papillary layer, has been destroyed. In a certain number of cases, variously estimated by the writers on the subject, but according to the average estimate in about one case out of three, this ulcer during its course is complicated by an acute adenitis of an inguinal gland, sometimes on each side, which may result in suppuration. This adenitis may be either sympathetic, like that in the axilla after a bruise of the finger, or it may be due to the actual absorption of the virus of the ulcer by the lymphatics. This virus is arrested at the first gland with which the lymphatic vessels connect, and there sets up its specific inflammation, resulting in an abscess, which opens, and the pus from it possesses the specific property (the power of reproducing a similar ulcer when inoculated) of the chancroid.

Such is a very brief description of this affection, which, it will readily be admitted, is one sui generis, having many marked individual characteristics, in the combination of which it differs from all other known forms of ulceration. From the anatomical situation of this ulcer, namely, on the external integument or mucous membrane, and the consequent ease with which it can be watched and studied, and from the fact of the possibility of its artificial reproduction by inoculation, it would be supposed that its history, course, pathology, and treatment would have been thoroughly investigated, and such is the case. Many able and careful observers have worked in this field, and the many inoculations made, either to settle the question of differential diagnosis between this disease and the syphilitic lesion, or during the investigation of the subject of syphilization, reaching in a case of Danielssen, of Christiania, the enormous number of four hundred on one patient, have resulted in a thorough knowledge of the ulcer from the moment of its first appearance to the end; and there are few subjects in medical science on which there is such perfect unanimity of opinion, with the exception of some differences in abstruse points of causation and specific nature which exist between the followers of the belief in the unity and those who believe in the duality of the virus, the former, however, being now reduced to a very small number of adherents.

With regard to treatment, even the believers in unity are in accord with the rest of the world, and the treatment universally taught is cauterization. By cauterization is not meant touching the ulcer with a crayon of nitrate of silver, but actual, destructive cauterization, for which purpose the whole arsenal of the strong acids, and caustic pastes, and even the actual cautery, have been recommended and used. 
The theory of this treatment is that, as the chancre secretes a specific virus and shows a specific tendency to progress by the ulcerative process, by destroying the base and edges thoroughly we substitute for the specific sore an ordinary solution of continuity, which will show a tendency to repair by the usual process of granulation. And such in point of fact is the result generally when the cauterization has been properly done ; when the resulting eschar comes off, the base looks healthy, and the process of granulation soon starts up. In some cases, however, the cauterization has to be repeated once or more before the ulcer loses its specific character. While all authorities admit that the chancroid as a rule is a self-limited disease, that is, that after a certain time it will, unless phagedæna sets in, stop spreading and gradually heal, it is claimed, and experiments seem to justify the clain, that the time required is from six weeks upwards, a period which is decidedly shortened by the caustic treatment.

Such being the case, what objections can be urged against this treatment?

In the first place it is quite painful, not so much so when there is one small chancre only; but when the ulcers are multiple, as they are apt to be, the pain may be very severe. It is true that we can use ether, but it must be remembered that giving ether in a hospital, where assistants and all the necessary adjuvants are at hand, is a very different thing from giving it in the physician's office. I hold that no patient should ever be etherized by a physician alone, and as most of these cases are treated in the office it would be necessary to call in an assistant. Much time is consumed, to say nothing of the liability to vomit and the very probable chance of the sphincters relaxing during or after the operation. Moreover, although we appreciate the great blessings of ether, it is often far from agreeable to take.

Secondly, the thorough cauterization of a chancroid is followed by considerable inflammatory action, and especially if it be on the prepuce, by odema and swelling. Now in those patients whose prepuces are long enough to cover the glans the cauterization of chancroids on the glans and on the inner fold of the prepuce will cause enough swelling to produce an artificial phymosis, and thus we shall lose the great advantage which we have in treating an external lesion to which topical treatment can be applied.

Thirdly, the inflammation produced by the caustic is apt to cause an induration of the surrounding tissues, which it is sometimes impossible to tell from the specific induration showing constitutional syphilitic infection, and in this way we obscure our means of diagnosis on a point which is of great importance to the patient.

Fourthly, the bubo which we find in a certain number of cases of chancroid may be either specific, caused by the actual absorption of the 
virus, or it may be what we call sympathetic, that is in some way due to the irritation at the seat of the ulcer. Now in the latter case it would seem that anything which increases the irritation must increase the chance of a bubo, and such I think is proved to be the fact by experience. I have no statistics to back the statement, but I feel sure that the percentage of co-existing buboes is greater in cases where thorough cauterization has been used than in those that have been treated in a milder way.

These four possible results of cauterization, namely, pain, swelling and phymosis, masking the diagnosis, and the greater frequency of sympathetic buboes, are surely, if correct, enough to induce us to employ some other treatment if we can find one that will be as effectual. Without giving exact statistics, I am confident that I am understating the number when $I$ say that during the last two and a half years I have treated, at the dispensary and in private practice, five hundred cases of chancroid, and that I am overstating when I say that in that time I may have used actual or destructive cauterization in a dozen cases.

The treatment that I have adopted, wherever possible, is the use of iodoform, and with very good results. I will merely speak of three cases, the data of which I take from my private case-book.

A patient who had been under treatment for five weeks for a chancroid, which had been burned with nitric acid about three times a week, and which nevertheless continued to increase slowly, was sent to see me for consultation. The ulcer had attained the size of a thumb-nail. In one week of dressing with iodoform it was entirely healed.

In another case of four weeks' duration there were seven chancroids about the glans and prepuce, one of which had eaten through the frænum. These also were all healed in one week.

The third case is under treatment at present, having reported on December $14 \mathrm{th}$. The patient came to me November $28 \mathrm{th}$, with a series of chancroids in the furrow of the glans, which practically amounted to one surrounding the whole glans, and with seven others on different parts of the prepuce and glans itself. They were of only four or five days' duration and had received no treatment whatever. There was so much inflammation and redness of the prepuce, which was quite long, that it was with great difficulty that it conld be retracted, and I am sure that any caustic applied would have made a phymosis that would have defied local treatment for at least a fortnight. On December 14th, the sixteenth day, the chancroids were all perfectly healthy granulating ulcers, and a swelling of an inguinal gland which had started up had gone down so much, that I removed the restriction with regard to exercise which I had placed on the patient. ${ }^{1}$

1 On the 21 st there was only onc spot about the size of a pin's head that had not healed, and the patient was discharged. 
I do not mean to say that these are three average cases, but I do mean that the duration of the disease has been less and the frequency of suppurating buboes decidedly less also than when I treated such cases by destructive cauterization. So sure am $I$ of this fact that $I$ have for some time been seeking to discover the why and the wherefore.

It does not seem possible that the large number of syphilographers in the last generation who have treated this subject experimentally should have accepted the fact that cauterization was necessary, when such was not the case. Begging the question with regard to the correctness of my conclusions, and assuming that $I$ am right in saying that at present, in the great majority of cases, cliancroids will heal in from one to three weeks withont cauterization, the other possible alternative is that the character and virulence of this lesion has changed. This opens a vast field for speculation and study, with regard to the nature of which I will merely offer the following hints. The experiments in syphilization have shown that by repeated re-inoculation of chancroidal virus it gradually loses its power to reproduce a chancroid, and that a time will come when the pus secreted from the resulting ulcer has nothing to distinguish it from ordinary pus. Now admitting that every chancroid is the result of contagion from another, in other words that every chancroid has a parent, and estimating the average life of a chancroid from the time of its birth to the period when it gives being to another at one month, which is certainly a long interval, - admitting these statements I say, any chancroid that we see to-day, must have been the result of two hundred and forty inoculations in the past twenty years.

Clerc takes the ground that the chancroid, although a local disease, owes its being to the fact of the syphilitic virus being inoculated in somebody who had already been syphilized, and under the circumstances it merely produces an ulcer, which, in turn, can only reproduce a local lesion.

Bumstead admits that there may be some truth in this theory. If there is, may not the fact that syphilis is quite prevalent among people who have chancroids explain the still greater modification of the virus, so that it may gradually lose its power of even producing a virulent local disease? But these are mere hints of the train of argument and study that might be followed. In point of fact $I$ have not seen, in the ten years during which $I$ have been in practice, one single case where a chancroid has caused a sufficient loss of tissue to leave a deformity, whereas I do quite often see cases where a considerable part of the glans or prepuce has been destroyed by chancroids which existed twenty or thirty years ago. But to return to the point which I wish to discuss. I have found that the great majority of cases of chancroid do well without destructive cauterization. I am aware that it will be said, and with truth, that some of these cases may not have been chancroids, but irri- 
tated abrasions, or herpetic ulcers, which would have got well if they had been let alone. Such cases, however, were certainly so few in number that they need not be taken into account. My attention was first called to the fact that chancroids would do well without cauterization by happening to have several cases under my care at the same time about three years ago, in which the existence of a phymosis, in some cases congenital, in others the result of inflammatory action, made it impossible to treat them by cauterization, and yet in time they all got well ; and in those cases where the phymosis subsided the loss of tissue was not noticeable. The time required was from four to eight weeks. From the amount of pus, swelling, etc., in three of the cases at least, I was in great doubt as to whether I should not slit up the prepuce in order to be able to cauterize, and thus put a stop to the spreading of the ulceration, and had the tide not turned when it did, should have done so in one case certainly. Since that time, I have not used cauterization in more than a dozen cases. With regard to the use of iodoform, I hardly dare to claim for it a specific action in causing chancroids to heal quickly. No one can be more on his guard against the "post hoc, propter hoc" line of reasoning, and I regret to say that my faith in specifics is very small, or rather confined to very few; but on the other hand, when I have seen sufficient proofs that certain drugs do act in a certain way, and there is nothing in what $I$ know of physiology, histology, or pathology to prove that they cannot act so, I believe that they do. For example, I never believed that iodide of potassium could cure an aneurism when the sac was once formed, nor that the chlorate of potash could cure an ovarian dropsy when the cyst was developed, although asserted by those to whose experience and judgment in most things $I$ defer; but I not only believe, but know, that a mercurial, properly administered, will hasten the clearing up of secondary syphilitic symptoms, that iodide of potassium will cause a gummy tumor to be absorbed without breaking down and suppurating, and that a mixed treatment of the two will heal in two weeks a venereal ulcer that has been slowly spreading for months. In the same way I know that iodoform will in most cases cause a chancroid to heal in a very short time. It is not the only thing under the use of which it will heal, as I have tried, with success, black wash, astringent lotions, mercurial ointment, etc., but the difference in time is very decided. Nor are chancroids the only ulcers on which iodoform seems to have a marked curative influence, as I was led to try it by having had an epithelial ulcer heal very quickly after this drug was used as a dressing. The cure in this case, however, was of course only temporary. It may be used either pure, as a powder, or made into an ointment ( $3 i-\xi i)$. The former is best where the ulcer is excavated, or the edges raised, as it can be sprinkled into the hollow places formed; the latter, when the 
ulcer is not deep, so that when spread on lint the ointment can touch the whole of the sore. There is only one objection to the use of iodoform, and that is quite a serious one, namely, the very powerful and penetrating odor with which it is endowed. Patients who are using it constantly hear the persons they are thrown in contact with complain of the gas leaking, etc. By care, this may be somewhat modified by putting dry lint over the powder when that is used, and by using a larger piece of lint than is necessary to cover the sore when the salve is the medium employed, and by carefully washing the hands after renewing the dressings. Although in most cases the use of iodoform will succeed, there may be need of having recourse to the classical treatment of cauterization, and in this case the selection of the caustic to be used is a matter of importance. For thorough and quick destruction of the tissues to which it is applied there is nothing so potent as pure bromine. It causes an instantaneous dry eschar, about a line in thickness, which on the third or fourth day falls off and discloses almost always a red healthy surface. It is, however, difficult to apply, as if too much is used it runs like liquid fire, shriveling up the tissues that it comes in contact with; moreover, the fumes are very irritating, and if inhaled while making the application will most effectually distract the surgeon's attention from his work to himself. It is best applied by means of a pipette or by a solid glass rod drawn out to a point. Another objection to its use is that it is not always easy to obtain. Fuming nitric acid I have found to be the next best caustic for this purpose, although the eschar formed is not nearly so thick. After applying it thoroughly, dry lint should be used as a dressing until the eschar comes off. Under no circumstances should nitrate of silver be used as a caustic in the treatment of chancroids. It can do no good and may do harm. Unfortunately, with many physicians the routine practice seems to be, if they see an abrasion, or a tear, or an ulcer on the penis, as to the nature of which they are in doubt, to touch it with caustic as a preliminary step, and wait for further development. Later, the solid stick may be used with great benefit, if after the chancroid has lost its specific character it has become a simple indolent ulcer, but the use of the nitrate then is to stimulate, not to destroy.

On reading over what I have hurriedly written, I see that I may perhaps have laid myself open to the charge of claiming originality in the views expressed. Nothing could be further from my thoughts. I have no doubt that many other observers have noticed that chancroids heal without the use of caustics, and I know that iodoform has been used in their treatment. Indeed, Bumstead mentions incidentally, concerning the subject of phagedænic chancroids, that he has used it with good effect, but nevertheless, as the fact remains that in all text-books cauterization is laid down as the rule, and as in the periodical literature that 
has come under my eye I have seen no other view advanced, and as I am convinced that in ninety cases out of a hundred, at the very least, it is not only unnecessary but injurious, I have attempted to give my grounds for this conviction.

\section{RECENT PROGRESS IN MEDICAL CHEMISTRY. ${ }^{1}$}

BY E. 8. WOOD, M. D.

Copper. - MM. Bergeron and L'Hôte ${ }^{2}$ have made a series of analyses for the purpose of settling the question as to the amount of copper normally present in the human body. About one half of the liver and one kidney were taken for analysis in each case. In two individuals seventeen years of age, copper was detected, although its amount was not estimated; in eleven persons aged from twenty-six to fifty-eight years, between 0.0007 and $0.0010 \mathrm{grm}$. was found in each case; and in one aged seventy-eight years $0.0015 \mathrm{grm}$. was detected. Copper was also found in six fotal livers. They conclude, therefore, that copper is always present in appreciable quantity in the liver and kidneys, and they do not think that the amount of copper in the whole liver and kidneys ever exceeds two and a half to three milligrammes, and in most cases does not reach two milligrammes.

These gentlemen made the analyses of the organs of the two females in the "Moreau" poisoning case, and found in the liver an amount of copper corresponding to $0.119 \mathrm{grm}$. of the sulphate of copper in one case, and to $0.084 \mathrm{grm}$. in the other. They stated, therefore, that since so large an amount of copper was found, and because the symptoms were not inconsistent with acute copper poisoning, and a careful postmortem examination failed to reveal a natural cause for death, a compound of copper must have been the cause of death.

I. M. V. Galippe ${ }^{3}$ takes exception to the above conclusions of Bergeron and L'Hôte, and considers that an expert has no right to state that death has been produced by copper poisoning when such small amounts as the above are found, since much larger quantities have been detected in cases in which death has been due to natural causes. Thus MM. Bourneville and Yvon ${ }^{4}$ found in the liver of an epileptic, who had during iife been treated with the ammonio-sulphate of copper, and had taken forty-three grm. of this compound, $0.295 \mathrm{grm}$. of metallic copper, which corresponds to $1.166 \mathrm{grm}$. of the sulphate of copper, although the patient had taken no copper compound for three months before death. Yvon found in the liver of another person, who had

1 Concluded from page 15.

2 Journal de Chimie médicale, March, 1875, page 115.

8 Étude toxicologique sur le Cuivre et ses Composés, Paris, 1875.

- Journal de Chimie médicale, May, 1875. 